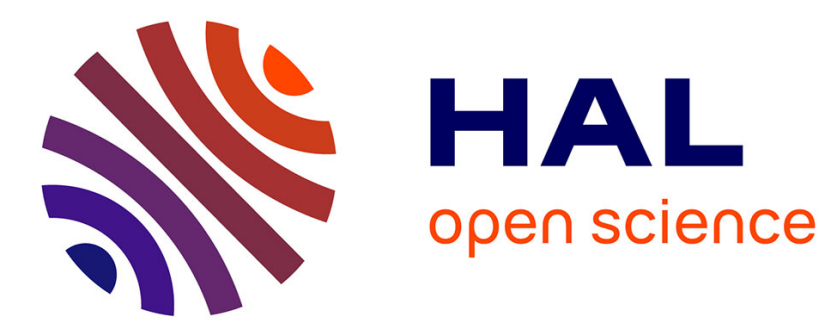

\title{
Optimal widely linear MVDR beamforming for noncircular signals
}

\author{
Pascal Chevalier, Jean-Pierre Delmas, Abdelkader Oukaci
}

\section{To cite this version:}

Pascal Chevalier, Jean-Pierre Delmas, Abdelkader Oukaci. Optimal widely linear MVDR beamforming for noncircular signals. ICASSP 2009: IEEE International Conference on Acoustics, Speech, and Signal Processing, Apr 2009, Taipei, Taiwan. pp.3573 - 3576, 10.1109/ICASSP.2009.4960398. hal-01361058

\section{HAL Id: hal-01361058 \\ https://hal.science/hal-01361058}

Submitted on 15 Dec 2020

HAL is a multi-disciplinary open access archive for the deposit and dissemination of scientific research documents, whether they are published or not. The documents may come from teaching and research institutions in France or abroad, or from public or private research centers.
L'archive ouverte pluridisciplinaire HAL, est destinée au dépôt et à la diffusion de documents scientifiques de niveau recherche, publiés ou non, émanant des établissements d'enseignement et de recherche français ou étrangers, des laboratoires publics ou privés. 


\section{OPTIMAL WIDELY LINEAR MVDR BEAMFORMING FOR NONCIRCULAR SIGNALS}

\author{
Pascal Chevalier \\ Thales-Communications, EDS/SPM \\ 92704 Colombes, France
}

\author{
Jean-Pierre Delmas, Abdelkader Oukaci \\ Telecom SudParis, UMR CNRS 5157 \\ 91011 Evry, France
}

\begin{abstract}
This paper introduces the optimal widely linear (WL) minimum variance distorsionless response (MVDR) beamformer for the reception of an unknown signal of interest (SOI) corrupted by potentially second order (SO) noncircular background noise and interference. The SOI, whose waveform is unknown, is assumed to be SO noncircular with arbitrary noncircular properties. In the steady state and for SO noncircular SOI and/or interference, this new WL beamformer, that is derived from an original orthogonal decomposition, is shown to always improve the performance of both the wellknown Capon's beamformer and a WL MVDR beamformer introduced recently in the literature. This optimal WL MVDR beamformer is first introduced and some of its performance are analyzed. Then, several adaptive implementations of this optimal WL beamformer are presented.
\end{abstract}

Index Terms - Noncircular, widely linear, interference, beamforming, MVDR beamforming

\section{INTRODUCTION}

Conventional beamforming approaches aim at finding a linear and time invariant (TI) complex filter $\mathbf{w}$, such that its output $y(t) \stackrel{\text { def }}{=} \mathbf{w}^{H} \mathbf{x}(t)$ corresponds to a SO estimate of a SOI coming from a particular direction and potentially corrupted by interference plus background noise, where $\mathbf{x}(t)$ is the vector of the complex envelopes of the signals observed at the output of the sensors. Although SO optimal for stationary observations [1], whose complex envelopes are necessarily SO circular [2], this conventional approach becomes suboptimal for nonstationary signals [1], omnipresent in radio communication contexts, whose complex envelope may also become SO noncircular [2] such as BPSK, ASK, MSK or GMSK signals for example. More precisely, for nonstationary observations, the optimal complex filters become time variant [3], and under some conditions of noncircularity [1], WL [4], i.e., of the form $y(t)=\mathbf{w}_{1}(t)^{H} \mathbf{x}(t)+\mathbf{w}_{2}(t)^{H} \mathbf{x}(t)^{*}$.

Very recently, a TI WL MVDR beamformer has been introduced and deeply analyzed in [5]. However, although more powerful than the Capon's beamformer for SO noncircular interference, this WL beamformer remains suboptimal for a
SO noncircular SOI, since it does not exploit the SO noncircularity of the latter. To overcome this limitation, the purpose of this paper is to introduce the optimal TI WL MVDR beamformer for the reception of an unknown SOI corrupted by potentially SO noncircular background noise and interference. The SOI, whose waveform is unknown, is assumed to be SO noncircular with arbitrary noncircular properties. This new WL MVDR beamformer, based on an original orthogonal decomposition, takes into account the potential SO noncircularity of both the SOI and interference. It is shown to always improve, in the steady state and for SO noncircular SOI and/or interference, the performance of both the well-known Capon's beamformer and the WL MVDR beamformer introduced in [5]. Several adaptive implementations of this new WL beamformer are then presented assuming that both the SO noncircularity and the steering vector of the SOI are not necessarily known.

\section{HYPOTHESES, DATA STATISTICS AND PROBLEM FORMULATION}

Let us consider an array of $N$ narrow-band sensors and denote by $\mathbf{x}(t)$ the vector of complex amplitudes of the signals at the output of these sensors. Each sensor is assumed to receive a SOI corrupted by a total noise (potentially composed of interference and background noise). Under these assumptions, the observation vector $\mathbf{x}(t)$ can be written as follows

$$
\mathbf{x}(t)=s(t) \mathbf{s}+\mathbf{n}(t),
$$

where $s(t)$ and $\mathbf{s}$ correspond to the complex envelope, assumed zero-mean and potentially SO noncircular, and the steering vector, such that its first component is equal to one, of the SOI respectively. The vector $\mathbf{n}(t)$ is the total noise vector, assumed zero-mean, potentially SO noncircular and statistically uncorrelated with $s(t)$.

The SO statistics of the noncircular observation $\mathbf{x}(t)$ are defined by

$$
\begin{aligned}
& \mathbf{R}_{x} \stackrel{\text { def }}{=}<\mathrm{E}\left[\mathbf{x}(t) \mathbf{x}(t)^{H}\right]>=\pi_{s} \mathbf{s s}^{H}+\mathbf{R}_{n}, \\
& \mathbf{C}_{x} \stackrel{\text { def }}{=}<\mathrm{E}\left[\mathbf{x}(t) \mathbf{x}(t)^{T}\right]>=\pi_{s} \gamma_{s} \mathbf{s s}^{T}+\mathbf{C}_{n},
\end{aligned}
$$

where $<$. $>$ denotes the time-averaging operation, with respect to the time index $t$, over the observation window, 
$\pi_{s} \stackrel{\text { def }}{=}<\mathrm{E}\left[|s(t)|^{2}\right]>$ is the time-averaged power of the SOI received by the first sensor, $\gamma_{s} \stackrel{\text { def }}{=}<\mathrm{E}\left[s(t)^{2}\right]>/<$ $\mathrm{E}\left[|s(t)|^{2}\right]>\stackrel{\text { def }}{=}\left|\gamma_{s}\right| e^{2 i \phi_{s}}$ such that $0 \leq\left|\gamma_{s}\right| \leq 1$, is the time-averaged SO noncircularity coefficient of the SOI, $\mathbf{R}_{n} \stackrel{\text { def }}{=}<\mathrm{E}\left[\mathbf{n}(t) \mathbf{n}(t)^{H}\right]>$ and $\mathbf{C}_{n} \stackrel{\text { def }}{=}<\mathrm{E}\left[\mathbf{n}(t) \mathbf{n}(t)^{T}\right]>$.

In order to introduce WL filters in the following, we define the extended observation vector by $\tilde{\mathbf{x}}(t) \stackrel{\text { def }}{=}\left[\mathbf{x}(t)^{T}, \mathbf{x}(t)^{H}\right]^{T}$ and using (1) we obtain

$$
\tilde{\mathbf{x}}(t)=s(t) \tilde{\mathbf{s}}_{1}+s(t)^{*} \tilde{\mathbf{s}}_{2}+\tilde{\mathbf{n}}(t) \stackrel{\text { def }}{=} \tilde{\mathbf{S}} \tilde{\mathbf{s}}(t)+\tilde{\mathbf{n}}(t),
$$

where $\tilde{\mathbf{n}}(t) \stackrel{\text { def }}{=}\left[\mathbf{n}(t)^{T}, \mathbf{n}(t)^{H}\right]^{T}, \tilde{\mathbf{s}}_{1} \stackrel{\text { def }}{=}\left[\mathbf{s}^{T}, \mathbf{0}_{N}^{T}\right]^{T}, \tilde{\mathbf{s}}_{2} \stackrel{\text { def }}{=}$ $\left[\mathbf{0}_{N}^{T}, \mathbf{s}^{H}\right]^{T}, \tilde{\mathbf{s}} \stackrel{\text { def }}{=}\left[\tilde{\mathbf{s}}_{1}, \tilde{\mathbf{s}}_{2}\right]$ and $\tilde{\mathbf{s}}(t) \stackrel{\text { def }}{=}\left[s(t), s(t)^{*}\right]^{T}$. The SO statistics of $\tilde{\mathbf{x}}(t)$ considered in this paper correspond to the time-averaged matrix $\mathbf{R}_{\tilde{x}} \stackrel{\text { def }}{=}<\mathrm{E}\left[\tilde{\mathbf{x}}(t) \tilde{\mathbf{x}}(t)^{H}\right]>$ given, under the previous assumptions, by $\mathbf{R}_{\tilde{x}}=\tilde{\mathbf{S}} \mathbf{R}_{\tilde{s}} \tilde{\mathbf{S}}^{H}+$ $\mathbf{R}_{\tilde{n}}$ where $\mathbf{R}_{\tilde{s}} \stackrel{\text { def }}{=}<\mathrm{E}\left[\tilde{\mathbf{s}}(t) \tilde{\mathbf{s}}(t)^{H}\right]>$ and where $\mathbf{R}_{\tilde{n}} \stackrel{\text { def }}{=}<$ $\mathrm{E}\left[\tilde{\mathbf{n}}(t) \tilde{\mathbf{n}}(t)^{H}\right]>$ can be written as

$$
\mathbf{R}_{\tilde{n}}=\left(\begin{array}{cc}
\mathbf{R}_{n} & \mathbf{C}_{n} \\
\mathbf{C}_{n}^{*} & \mathbf{R}_{n}^{*}
\end{array}\right)
$$

We consider a TI WL spatial filter $\widetilde{\mathbf{w}} \stackrel{\text { def }}{=}\left[\mathbf{w}_{1}^{T}, \mathbf{w}_{2}^{T}\right]^{T}$ whose output is defined by

$$
y(t)=\widetilde{\mathbf{w}}^{H} \tilde{\mathbf{x}}(t)=s(t) \widetilde{\mathbf{w}}^{H} \tilde{\mathbf{s}}_{1}+s(t)^{*} \widetilde{\mathbf{w}}^{H} \tilde{\mathbf{s}}_{2}+\widetilde{\mathbf{w}}^{H} \tilde{\mathbf{n}}(t) .
$$

The problem addressed in this paper is to find the TI WL MVDR spatial filter $\widetilde{\mathbf{w}}$ which generates the best SO estimate of the SOI $s(t)$, whose waveform and content are unknown.

\section{OPTIMAL WL BEAMFORMER NOT TAKING INTO ACCOUNT SOI NONCIRCULARITY}

As $\gamma_{s}$ is unknown, a first philosophy is to built a WL MVDR beamformer which does not require the knowledge or the estimation of this coefficient. In this context, an efficient way to generate, in the output $y(t)$, a non-null SOI without any distortion whatever the correlation between $s(t)$ and $s(t)^{*}$, is to minimize the time-averaged output power $\widetilde{\mathbf{w}}{ }^{H} \mathbf{R}_{\tilde{x}} \widetilde{\mathbf{w}}$ under the constraints:

$$
\widetilde{\mathbf{w}}^{H} \tilde{\mathbf{s}}_{1}=1 \text { and } \widetilde{\mathbf{w}}^{H} \tilde{\mathbf{s}}_{2}=0 .
$$

The TI WL filter solution to this problem is the so-called WL $\mathrm{MVDR}_{1}$ beamformer introduced and analyzed in [5] and defined by

$$
\widetilde{\mathbf{w}}_{\mathrm{MVDR}_{1}}=\mathbf{R}_{\tilde{x}}^{-1} \tilde{\mathbf{S}}\left[\tilde{\mathbf{S}}^{H} \mathbf{R}_{\tilde{x}}^{-1} \tilde{\mathbf{S}}\right]^{-1} \mathbf{f},
$$

with $\mathbf{f} \stackrel{\text { def }}{=}[1,0]^{T}$. For a SO circular noise vector $\mathbf{n}(\mathrm{t})$, this WL beamformer reduces to the well-known Capon's MVDR beamformer defined by

$$
\mathbf{w}_{\text {Capon }} \stackrel{\text { def }}{=}\left(\mathbf{s}^{H} \mathbf{R}_{x}^{-1} \mathbf{s}\right)^{-1} \mathbf{R}_{x}^{-1} \mathbf{s},
$$

In the presence of SO noncircular interference, the WL $\mathrm{MVDR}_{1}$ beamformer outperforms the Capon's beamformer, in terms of output SINR and number of interference sources to be processed. Moreover, all the adaptive algorithms developed to implement the Capon's beamformer can be easily extended to this WL MVDR 1 beamformer.

Finally, let us point out that as the WL MVDR 1 beamformer does not exploit the potential SO noncircularity of the SOI, it does not allow for single antenna interference cancellation (SAIC) [6] in the presence of both rectilinear SOI and interference. The WL MVDR beamformer introduced in the next section aims precisely at overcoming this limitation by taking into account the potential SO noncircularity of the SOI.

\section{OPTIMAL WL MVDR BEAMFORMER TAKING INTO ACCOUNT SOI NONCIRCULARITY}

For $\gamma_{s} \neq 0, s(t)^{*}$ is correlated with $s(t)$ and contains both a SOI and an interference component. To compute the SOI component of $s(t)^{*}$, let us consider the Hilbert space of random processes having a finite time-averaged power and fitted with the inner product $(u(t), v(t)) \stackrel{\text { def }}{=}<\mathrm{E}\left[u(t) v(t)^{*}\right]>$. It is then easy to compute the orthogonal projection of $s(t)^{*}$ on $s(t)$ for the previous inner product. This gives the following orthogonal decomposition in this space

$$
s(t)^{*}=\gamma_{s}^{*} s(t)+\left[\pi_{s}\left(1-\left|\gamma_{s}^{2}\right|\right)\right]^{1 / 2} s^{\prime}(t),
$$

with $<\mathrm{E}\left[s(t) s^{\prime}(t)^{*}\right]>=0$ and $<\mathrm{E}\left[\left|s^{\prime}(t)\right|^{2}\right]>=1$. Decomposition (6) shows that, for a given time-averaged useful input power $\pi_{s}$, the time-averaged power of the desired signal component of $s(t)^{*}$ is equal to $\pi_{s}\left|\gamma_{s}\right|^{2}$ and increases with $\left|\gamma_{s}\right|$. In particular for a rectilinear SOI for the receiver (BPSK, ASK), $\gamma_{s}=e^{2 i \phi_{s}}, s(t)^{*}=e^{-2 i \phi_{s}} s(t)$ and $s(t)^{*}$ totally corresponds to the SOI, whereas for a SO circular SOI (e.g., QPSK), $\gamma_{s}=0, s(t)^{*}=\pi_{s}^{1 / 2} s^{\prime}(t)$, and $s(t)^{*}$ totally corresponds to an interference for the SOI. Using (6) in (2), we obtain

$$
\begin{gathered}
\tilde{\mathbf{x}}(t)=s(t) \underbrace{\left(\tilde{\mathbf{s}}_{1}+\gamma_{s}^{*} \tilde{\mathbf{s}}_{2}\right)}_{\tilde{\mathbf{s}}_{\gamma}}+\underbrace{s^{\prime}(t)\left[\pi_{s}\left(1-\left|\gamma_{s}^{2}\right|\right)\right]^{1 / 2} \tilde{\mathbf{s}}_{2}+\tilde{\mathbf{n}}(t)}_{\tilde{\mathbf{n}}_{\gamma}(t)} \\
\stackrel{\text { def }}{=} s(t) \tilde{\mathbf{s}}_{\gamma}+\tilde{\mathbf{n}}_{\gamma}(t)
\end{gathered}
$$

where $\tilde{\mathbf{s}}_{\gamma}$ and $\tilde{\mathbf{n}}_{\gamma}(t)$ are the equivalent extended steering vector of the SOI, which now depends on $\gamma_{s}$, and the global noise vector respectively for the extended observation vector $\tilde{\mathbf{x}}(t)$. Using (7) into (3), we finally obtain

$$
y(t)=\widetilde{\mathbf{w}}^{H} \tilde{\mathbf{x}}(t)=s(t) \widetilde{\mathbf{w}}^{H} \tilde{\mathbf{s}}_{\gamma}+\widetilde{\mathbf{w}}^{H} \tilde{\mathbf{n}}_{\gamma}(t) .
$$

From decomposition (8), we deduce that the optimal WL MVDR beamformer, which optimally exploits the parameters $\mathbf{s}$ and $\gamma_{s}$, corresponds to the WL filter $\widetilde{\mathbf{w}}$ which minimizes the 
time-averaged output power $\widetilde{\mathbf{w}}^{H} \mathbf{R}_{\tilde{x}} \widetilde{\mathbf{w}}$, under the following constraint:

$$
\widetilde{\mathbf{w}}^{H} \widetilde{\mathbf{s}}_{\gamma}=1 .
$$

This WL MVDR beamformer, called $\mathrm{MVDR}_{2}$ in the following, is defined by

$\widetilde{\mathbf{w}}_{\mathrm{MVDR}_{2}} \stackrel{\text { def }}{=}\left[\tilde{\mathbf{s}}_{\gamma}^{H} \mathbf{R}_{\tilde{x}}^{-1} \tilde{\mathbf{s}}_{\gamma}\right]^{-1} \mathbf{R}_{\tilde{x}}^{-1} \tilde{\mathbf{s}}_{\gamma}=\left[\tilde{\mathbf{s}}_{\gamma}^{H} \mathbf{R}_{\tilde{n}_{\gamma}}^{-1} \tilde{\mathbf{s}}_{\gamma}\right]^{-1} \mathbf{R}_{\tilde{n}_{\gamma}}^{-1} \tilde{\mathbf{s}}_{\gamma}$,

where the time-averaged first correlation matrix $\mathbf{R}_{\tilde{n}_{\gamma}}$ of $\tilde{\mathbf{n}}_{\gamma}(t)$ can be written from (7) as

$$
\mathbf{R}_{\tilde{n}_{\gamma}}=\left[\pi_{s}\left(1-\left|\gamma_{s}^{2}\right|\right)\right] \tilde{\mathbf{s}}_{2} \tilde{\mathbf{s}}_{2}^{H}+\mathbf{R}_{\tilde{n}} .
$$

This WL beamformer may thus be implemented from the knowledge or the estimation of $\left(\mathbf{s}, \gamma_{s}\right)$ and either from the observations $\mathbf{x}(t)$ or from both a total noise alone reference $\mathbf{n}(t)$, when such a reference is available, and the knowledge of $\pi_{s}$. We verify that the beamformer $\widetilde{\mathbf{w}}_{\mathrm{MVDR}_{2}}$ fits the Capon's beamformer (5) when both the SOI and the total noise are SO circular $\left(\gamma_{s}=0, \mathbf{C}_{n}=\mathbf{O}\right)$. Finally note that for a rectilinear SOI $\left(\left|\gamma_{s}\right|=1\right)$, this WL beamformer corresponds to the WL spatial matched filter introduced in [6].

Equation (8) clearly displays the SOI and the global noise uncorrelated parts of $y(t)$. It is then straightforward to introduce the SINR at the output of a WL filter $\widetilde{\mathbf{w}}$ defined by

$$
\operatorname{SINR}[\widetilde{\mathbf{w}}] \stackrel{\text { def }}{=} \frac{\pi_{s}\left|\widetilde{\mathbf{w}}^{H} \tilde{\mathbf{s}}_{\gamma}\right|^{2}}{\widetilde{\mathbf{w}}^{H} \mathbf{R}_{\tilde{n}_{\gamma}} \widetilde{\mathbf{w}}} .
$$

It is then easy to prove that $\widetilde{\mathbf{w}}_{\mathrm{MVDR}_{2}}$ maximizes this SINR and is also proportional to $\widetilde{\mathbf{w}}_{\mathrm{MMSE}}$ which minimizes the mean square error

$$
\operatorname{MSE}[\widetilde{\mathbf{w}}] \stackrel{\text { def }}{=}<\mathrm{E}\left[\left|s(t)-\widetilde{\mathbf{w}}^{H} \tilde{\mathbf{x}}(t)\right|^{2}\right]>.
$$

\section{PERFORMANCE}

\subsection{SINR and MSE}

From expressions (4), (5) and (10), it is possible to compute the value of the SINR (11) at the output of the Capon, WL $\mathrm{MVDR}_{1}$ and WL $\mathrm{MVDR}_{2}$ beamformers respectively. It is easy to verify that these values are given by

$$
\begin{aligned}
\operatorname{SINR}_{\text {Capon }} & =\pi_{s} \mathbf{s}^{H} \mathbf{R}_{n}^{-1} \mathbf{s}, \\
\mathrm{SINR}_{\mathrm{MVDR}_{1}} & =\frac{\pi_{s}}{\mathbf{f}^{T}\left[\tilde{\mathbf{S}}^{H} \mathbf{R}_{\tilde{n}}^{-1} \tilde{\mathbf{S}}\right]^{-1} \mathbf{f}}, \\
\mathrm{SINR}_{\mathrm{MVDR}_{2}} & =\pi_{s} \tilde{\mathbf{s}}_{\gamma}^{H} \mathbf{R}_{n_{\gamma}}^{-1} \tilde{\mathbf{s}}_{\gamma} .
\end{aligned}
$$

For a given source scenario, it is easy to compare these values of SINR without any particular computations. Indeed, $\mathbf{w}_{\text {Capon }}, \widetilde{\mathbf{w}}_{\mathrm{MVDR}_{1}}$ and $\widetilde{\mathbf{w}}_{\mathrm{MVDR}_{2}}$, all minimize the time-averaged output power $\widetilde{\mathbf{w}}^{H} \mathbf{R}_{\tilde{x}} \widetilde{\mathbf{W}}$ but under different constraints that satisfy

$$
\begin{aligned}
\{\widetilde{\mathbf{w}} & \left.=\left[\mathbf{w}_{1}^{T}, \mathbf{0}_{N}^{T}\right]^{T} ; \mathbf{w}_{1}^{H} \mathbf{s}=1\right\} \\
& \subset\left\{\widetilde{\mathbf{w}} ; \widetilde{\mathbf{w}}^{H} \widetilde{\mathbf{s}}_{1}=1 \text { and } \widetilde{\mathbf{w}}^{H} \widetilde{\mathbf{s}}_{2}=0\right\} \subset\left\{\widetilde{\mathbf{w}} ; \widetilde{\mathbf{w}}^{H} \widetilde{\mathbf{s}}_{\gamma}=1\right\} .
\end{aligned}
$$

Consequently, the inclusion principle implies that generally

$$
\mathrm{SINR}_{\text {Capon }} \leq \mathrm{SINR}_{\mathrm{MVDR}_{1}} \leq \mathrm{SINR}_{\mathrm{MVDR}_{2}} .
$$

Using decomposition (8) and the general expressions (11) and (12) of the SINR and MSE, the following relation between the MSE and SINR criteria is easily proved.

$$
\operatorname{MSE}[\widetilde{\mathbf{w}}]=\pi_{s}\left(\left|1-\widetilde{\mathbf{w}}^{H} \tilde{\mathbf{S}}_{\gamma}\right|^{2}+\frac{\left|\widetilde{\mathbf{w}}^{H} \tilde{\mathbf{s}}_{\gamma}\right|^{2}}{\operatorname{SINR}[\widetilde{\mathbf{w}}]}\right) .
$$

This relation is interesting since it shows that the WL filter $\widetilde{\mathbf{w}}$ which minimizes MSE[ $\widetilde{\mathbf{w}}]$ under the constraint $\widetilde{\mathbf{w}}^{H} \tilde{\mathbf{S}}_{\gamma}=1$, is also the WL filter which maximizes $\operatorname{SINR}[\widetilde{\mathbf{w}}]$ under the same constraint, which finally corresponds to $\widetilde{\mathbf{w}}_{\mathrm{MVDR}_{2}}$. This gives in particular a physical meaning of both decomposition (6) and SINR criterion. Finally, note that $\widetilde{\mathbf{w}}_{\mathrm{MMSE}}$ and $\widetilde{\mathbf{w}}_{\mathrm{MVDR}_{2}}$ that are collinear, generate the same output SINR, but not the same time-averaged MMSE. Indeed, we straightforwardly prove that

whereas

$$
\operatorname{MSE}\left[\widetilde{\mathbf{w}}_{\mathrm{MMSE}}\right]=\frac{\pi_{s}}{1+\mathrm{SINR}_{\mathrm{MVDR}_{2}}},
$$

$$
\operatorname{MSE}\left[\widetilde{\mathbf{w}}_{\mathrm{MVDR}_{2}}\right]=\frac{1}{\mathbf{s}_{\gamma}^{H} \mathbf{R}_{\tilde{n}_{\gamma}}^{-1} \mathbf{s}_{\gamma}}=\frac{\pi_{s}}{\operatorname{SINR}_{\mathrm{MVDR}_{2}}},
$$

which is greater than MSE $\left[\widetilde{\mathbf{w}}_{\mathrm{MMSE}}\right]$ and which tends toward $\mathrm{MSE}\left[\widetilde{\mathbf{w}}_{\mathrm{MMSE}}\right]$ as $\mathrm{SINR}_{\mathrm{MVDR}_{2}} \gg 1$.

\subsection{Processing capacity}

Let us now consider the particular case where the total noise $\mathbf{n}(t)$ is composed of $P$ potentially SO noncircular interference sources and a background SO circular noise $\mathbf{n}^{\prime}(t)$. Under these assumptions, the total noise vector $\mathbf{n}(t)$ is given by $\mathbf{n}(t)=\sum_{p=1}^{P} j_{p}(t) \mathbf{j}_{p}+\mathbf{n}^{\prime}(t)$, where $j_{p}(t)$ and $\mathbf{j}_{p}$ denote the waveform and the steering vector of interference $p$ respectively. $\left(j_{p}(t)\right)_{p=1, \ldots, P}$ and $\mathbf{n}^{\prime}(t)$ are supposed uncorrelated. In a similar way as for the SOI, $j_{p}(t)^{*}$ can be decomposed as follows

$$
j_{p}(t)^{*}=\gamma_{p}^{*} j_{p}(t)+\left[\pi_{p}\left(1-\left|\gamma_{p}^{2}\right|\right)\right]^{1 / 2} j_{p}^{\prime}(t)
$$

where $\pi_{p}, \gamma_{p}$ and $j_{p}^{\prime}(t)$ are the power, the SO noncircularity coefficient and the noise part of $j_{p}(t)$, defined in a similar way as for the SOI. Consequently, the global noise $\tilde{\mathbf{n}}_{\gamma}(t)$ defined in (7) can be written as (16) where $\tilde{\mathbf{j}}_{p, \gamma} \stackrel{\text { def }}{=} \tilde{\mathbf{j}}_{p_{1}}+\gamma_{p}^{*} \tilde{\mathbf{j}}_{p_{2}}$ with $\tilde{\mathbf{j}}_{p_{1}} \stackrel{\text { def }}{=}\left(\mathbf{j}_{p}^{T}, \mathbf{0}_{N}^{T}\right)^{T}, \tilde{\mathbf{j}}_{p_{2}} \stackrel{\text { def }}{=}\left(\mathbf{0}_{N}^{T}, \mathbf{j}_{p}^{H}\right)^{T}$

$$
\begin{aligned}
& \tilde{\mathbf{n}}_{\gamma}(t)=s^{\prime}(t)\left[\pi_{s}\left(1-\left|\gamma_{s}\right|\right)\right]^{1 / 2} \tilde{\mathbf{s}}_{2} \\
& \quad+\sum_{p=1}^{P}\left(j_{p}(t) \tilde{\mathbf{j}}_{p, \gamma}+j_{p}^{\prime}(t)\left[\pi_{p}\left(1-\left|\gamma_{p}^{2}\right|\right)\right]^{1 / 2} \tilde{\mathbf{j}}_{p_{2}}\right)+\tilde{\mathbf{n}}^{\prime}(t) .
\end{aligned}
$$

We deduce from this decomposition, that each interference $p$ which is non rectilinear $\left(\left|\gamma_{p}\right| \neq 1\right)$ generates two uncorrelated 
components in the global noise $\tilde{\mathbf{n}}_{\gamma}(t)$, whereas a rectilinear interference $\left(\left|\gamma_{p}\right|=1\right)$, only generates one component. In a same way, a non rectilinear SOI generates one interference, whereas a rectilinear SOI does not generate any component in $\tilde{\mathbf{n}}_{\gamma}(t)$.

From this result, we easily deduce that the maximal number of interference that may be processed by the WL $\mathrm{MVDR}_{2}$ beamformer is defined by $P_{\max }=2(N-1)-P_{\mathrm{nr}}+\delta\left(1-\left|\gamma_{s}\right|\right)$, where $P_{\mathrm{nr}}$, such that $P_{\mathrm{nr}} \leq P_{\max }$, is the number of nonrectilinear interference and $\delta$ is the Kronecker symbol. In particular, for a rectilinear SOI, the WL MVDR 2 may reject up to $2 N-1$ rectilinear interference and has the ability to perform SAIC $\left(P_{\max }=1\right.$ for $\left.N=1\right)$, whereas for nonrectilinear SOI $P_{\max }=2(N-1)$.

\subsection{Illustrative examples}

To briefly illustrate (13) and the interest of $\widetilde{\mathbf{w}}_{\mathrm{MVDR}_{2}}$, we consider an array of $N=2$ sensors equispaced half a wavelength apart and we assume that the SOI, assumed to be either BPSK or QPSK, is corrupted by a BPSK interference plus a SO circular background noise. The direction of arrival (DOA) $\theta_{s}$, the phase $\phi_{s}$ and the SNR of the SOI are such that $\theta_{s}=0^{\circ}$, $\phi_{s}=0^{\circ}$ and SNR $=10 \mathrm{~dB}$, whereas the SNR of the interference, called Interference to noise ratio (INR), is such that INR $=20 \mathrm{~dB}$. Under these assumptions, Fig. 1 that exhibits the SINR as a function of the interference DOA, shows an increasing $\mathrm{SINR}_{\mathrm{MVDR}_{2}}$ with respect to $\mathrm{SINR}_{\mathrm{MVDR}_{1}}$ as the phase diversity $\phi$ between the BPSK SOI and the interference increases, due to the optimal exploitation of $\gamma_{s}$ by $\widetilde{\mathbf{w}}_{\mathrm{MVDR}_{2}}$. We also note the better performance obtained for a BPSK SOI with respect to a QPSK one, despite the fact that the performance of the latter remains better than those at the output of $\mathrm{MVDR}_{1}$.

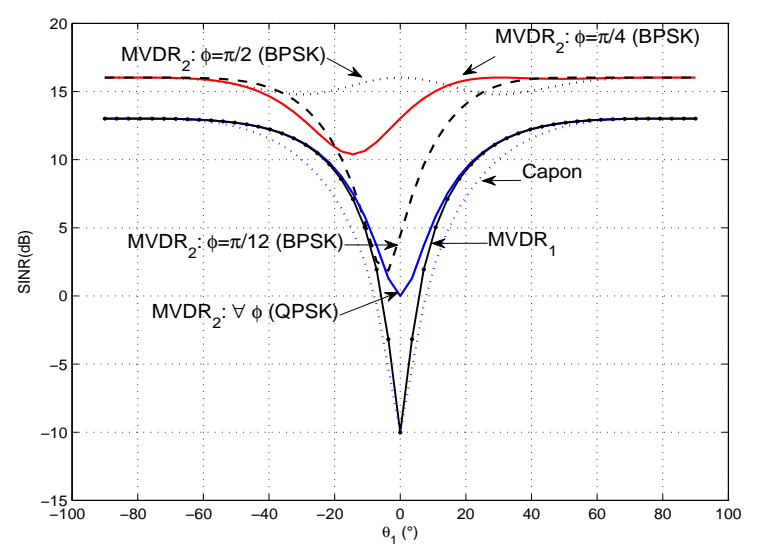

Fig.1 SINR Capon, $_{\text {SINR }}$ MVDR $_{1}$ and SINR $_{M V D R}$ as a function of the interference DOA $\theta_{1}$ for both BPSK and QPSK SOI

\section{IMPLEMENTATIONS}

When $\mathbf{s}$ and $\gamma_{s}$ are known, the WL MVDR 2 beamformer can be estimated from $K$ snapshots $\mathbf{x}\left(k T_{s}\right)$ by the sample matrix inverse (SMI) approach consisting to replace the statistics $\mathbf{R}_{\tilde{x}}$ by the empirical estimate $\widehat{\mathbf{R}}_{\tilde{x}} \stackrel{\text { def }}{=} \frac{1}{K} \sum_{k=1}^{K} \tilde{\mathbf{x}}\left(k T_{s}\right) \tilde{\mathbf{x}}\left(k T_{s}\right)^{H}$ in (10), where $T_{s}$ is the sample period. Note that the SMI implementation of the $\mathrm{WL} \mathrm{MVDR}_{1}$ and $\mathrm{MVDR}_{2}$ beamformers have the same order of complexity.

When both $\mathbf{s}$ and $\gamma_{s}$ are unknown, the extended steering vector $\tilde{\mathbf{s}}_{\gamma}$ can be estimated using a training sequence if available, or a blind identification approach. With a training sequence, $\tilde{\mathbf{s}}_{\gamma}$ can be estimated by the LS estimate $\widehat{\widetilde{\mathbf{s}}}_{\gamma}=\widehat{r}_{\tilde{s}}^{-1} \widehat{\mathbf{R}}_{\tilde{x}, s}$ derived from decomposition (7), with $\widehat{r}_{\widetilde{s}} \stackrel{\text { def }}{=}$ $\frac{1}{K} \sum_{k=1}^{K}\left|s\left(k T_{s}\right)\right|^{2}$ and $\widehat{\mathbf{R}}_{\tilde{x}, s} \stackrel{\text { def }}{=} \frac{1}{K} \sum_{k=1}^{K} \tilde{\mathbf{x}}\left(k T_{s}\right) s^{*}\left(k T_{s}\right)$. This gives an estimate $\widehat{\widetilde{\mathbf{w}}}_{\mathrm{MVDR}_{2}}=\left[\widehat{\widetilde{\mathbf{S}}}_{\gamma}^{H} \widehat{\mathbf{R}}_{\tilde{x}}^{-1} \widehat{\widetilde{\mathbf{S}}}_{\gamma}\right]^{-1} \widehat{\mathbf{R}}_{\tilde{x}}^{-1} \widehat{\widetilde{\mathbf{S}}}_{\gamma}$ which is proportional to the usual MMSE estimate $\widehat{\mathbf{R}}_{\tilde{x}}^{-1} \widehat{\mathbf{R}}_{\tilde{x}, s}$.

When a training sequence is not a priori available, $\tilde{\mathbf{s}}_{\gamma}$ can be blindly estimated (up to a source ambiguity) from a blind identification approach, such as the JADE algorithm [7], applied to the extended snapshots $\tilde{\mathbf{x}}\left(k T_{s}\right)$. Note that this approach is only valid for a rectilinear SOI. Fig. 2 shows the variations of the SINR at the output of the 3 previous estimated filters as a function of the number of snapshots for the scenario of Fig.1, for a BPSK SOI and for $\phi=90^{\circ}$ and $\theta_{1}=45^{\circ}$. Note the fast convergence of the 3 estimated filters.

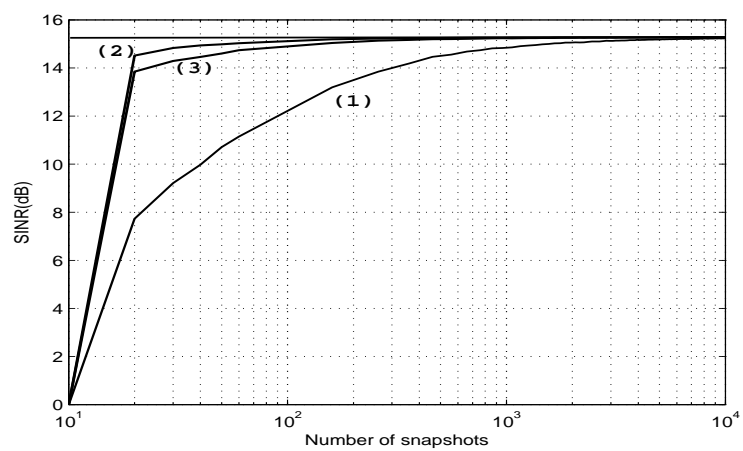

Fig.2 SINR given by the $\mathrm{MVDR}_{2}$ beamformer estimated by (1) extended SMI, (2) training sequence and (3) JADE approaches as a function of $K$.

\section{REFERENCES}

[1] P. Chevalier, "Optimal array processing for nonstationary signals", Proc. ICASSP, pp. 2868-2871, Atlanta (USA), May 1996.

[2] B. Picinbono, "On Circularity", IEEE Trans. Signal Process., vol. 42, no. 12, pp. 3473-3482, Dec 1994.

[3] W.A. Gardner, "Cyclic Wiener filtering: theory and method", IEEE Trans. on Com., vol 41, no. 1, pp. 151-163, Jan. 1993.

[4] B. Picinbono and P. Chevalier, "Widely linear estimation with complex data", IEEE Trans. Signal Process., vol. 43, no. 8, pp. 2030-2033, Aug. 1995.

[5] P. Chevalier and A. Blin, ’Widely linear MVDR beamformers for the reception of an unknown signal corrupted by noncircular interferences", IEEE Trans. Signal Process., vol. 55, no. 11, pp. 5323-5336, Nov 2007.

[6] P. Chevalier and F. Pipon, "New Insights into optimal widely linear array receivers for the demodulation of BPSK, MSK and GMSK signals corrupted by noncircular interferences - Application to SAIC', IEEE Trans. Signal Process., vol. 54, no. 3, pp. 870-883, March 2006.

[7] J.F. Cardoso and A. Souloumiac, "Blind beamforming for nonGaussian signals", IEE Proceedings- $F$, vol. 140, no. 6, pp. 362370, Dec. 1993. 